\title{
Lumen
}

Selected Proceedings from the Canadian Society for Eighteenth-Century Studies

\section{Les « Nocturnes » de Robert Challe}

\section{Jacques Cormier}

Volume 27, 2008

North America at the Crossroads of European Cultures in the Eighteenth Century

L’Amérique du Nord au Carrefour des cultures au XVIII ${ }^{\mathrm{e}}$ siècle

URI : https://id.erudit.org/iderudit/1012054ar

DOI : https://doi.org/10.7202/1012054ar

Aller au sommaire du numéro

Éditeur(s)

Canadian Society for Eighteenth-Century Studies / Société canadienne d'étude du dix-huitième siècle

ISSN

1209-3696 (imprimé)

1927-8284 (numérique)

Découvrir la revue

Citer cet article

Cormier, J. (2008). Les « Nocturnes » de Robert Challe. Lumen, 27, 109-136.

https://doi.org/10.7202/1012054ar

Copyright $@$ Canadian Society for Eighteenth-Century Studies / Société canadienne d'étude du dix-huitième siècle, 2009
Ce document est protégé par la loi sur le droit d'auteur. L'utilisation des services d'Érudit (y compris la reproduction) est assujettie à sa politique d'utilisation que vous pouvez consulter en ligne.

https://apropos.erudit.org/fr/usagers/politique-dutilisation/ 


\section{Les «Nocturnes» de Robert Challe}

En dépit de ses séjours au Canada et de son voyage aux Indes, Challe romancier est resté un piéton de Paris, essentiellement du Paris nocturne. Chez lui - comme chez Restif de la Bretonne qui, au moment où il rédige les Nuits de Paris, se souvient d'ailleurs d'avoir lu Les Illustres Françaises ${ }^{1}$ - , la nuit s'associe avant tout à Paris, ${ }^{2}$ et les personnages du grand roman challien arpentent souvent la capitale plongée dans l'obscurité. L'écrivain multiplie les observations qui renvoient à des détails précis. Dans nos villes éclairées $a$ giorno, nous ne savons plus ce que pouvait être, pour nos aïeux, l'expérience de la nuit. Challe, lui, vivait en un temps où les villes n'étaient pas éclairées, ou du moins l'époque dans laquelle il situe l'action de son roman se réfère à celle qui précède l'éclairage public: les années de son enfance, où les rues de Paris étaient obscures. ${ }^{3}$ Comme l'a signalé Marie-Christine Veneau, ${ }^{4}$ on

1 Les références aux Illustres Françaises renvoient à l'édition procurée par Frédéric Deloffre et Jacques Cormier, Genève, Droz, 1991, que nous désignons dans les notes par l'abréviation $I F$.

2 Pas exclusivement : à la fin des Illustres Françaises, la mort de Gallouin se produit de nuit, mais au cœur de la forêt de Brocéliande.

3 Les premiers essais d'éclairage public se firent en 1667. La mesure fut étendue par La Reynie qui établit un véritable éclairage public en 1668 : «Toutes les rues furent éclairées la nuit de lanternes qui contribuèrent à l'ornement aussi bien qu'à la sûreté ; et le guet à pied et à cheval, distribué dans tous les quartiers de la Ville, acheva d'en bannir les filous qui ne pouvaient plus s'y cacher impunément». (Dorival, Abrégé chronologique de l'Histoire de France sous les règnes de Louis XIII et de Louis XIV pour servir de suite à celui de François de Mezeray, Amsterdam, Pierre Mortier, 1735, t. II, p. 218).

4 Marie-Christine Veneau, «Challe et Marivaux : vieilles ou nouvelles folies romanesques", dans Jacques Cormier, Jan Herman et Paul Pelckmans (études réunies et présentées par), Robert Challe : Sources et héritages, Louvain, Peeters, 2003, p. 71-85, plus particulièrement les p. 81-82 et la n. 45 . 
ne trouve plus le même type de notations dans les romans de Marivaux, né en 1688, soit près de trente ans après Challe.

La façon dont la présence de la nuit est exploitée dans Les Illustres Françaises et le rôle que l'écrivain lui accorde se modifient au cours du roman. Dans les cinq premières histoires, la nuit est le cadre d'activités diverses, auxquelles elle donne parfois un relief saisissant. Dans les sixième et septième histoires, la nuit devient un élément actif de l'action romanesque dans la mesure où elle étend son ombre inquiétante sur les personnages ; l'obscurité dans laquelle se déroulent les événements importants fait écho aux états d'âmes des protagonistes: obscurité et nuit de l'âme y vont de pair. Mais on ne trouve pas dans l'œuvre romanesque de Robert Challe de place pour le rêve, ${ }^{5}$ pas plus qu'on n'y trouve de contemplation de la nature débouchant sur un contact fusionnel avec la poésie de la nuit, ce qu'on rencontrera en revanche occasionnellement dans le Journal de voyage. ${ }^{6}$

5 Cinquante ans plus tard, dans Julie ou la Nouvelle Hélö̈se, Rousseau, prêtant la parole à Saint-Preux, enregistre les manifestations d'un cauchemar lié au retour dans un même lieu d'un individu qui perçoit les modifications subies par son histoire personnelle alors même qu'il observe la permanence du lieu : «En entrant dans la chambre qui m'étoit destinée, je la reconnus pour la même que j'avois occupée autrefois en allant à Sion. A cet aspect je sentis une impression que j'aurois peine à vous rendre. J'en fus si vivement frappé, que je crus redevenir à l'instant tout ce que j'étois alors : Dix années s'effacèrent de ma vie, \& tous mes malheurs furent oubliés. Hélas! Cette erreur fut courte, \& le second instant me rendit plus accablant le poids de toutes mes anciennes peines. [...] Je me couchai dans ces tristes idées. Elles me suivirent durant mon sommeil, \& le remplirent d'images funèbres. [...] Un rêve surtout, le plus cruel de tous, s'obstinoit à me poursuivre, \& de phantôme en phantôme, toutes leurs apparitions confuses finissoient toujours par celui-là. [...] Je crus voir la digne mere de votre amie dans un lit, expirant, \& sa fille à genoux devant elle fondant en larmes, baisant ses mains, \& recueillant ses derniers soupirs. Je revis cette scène [...]. Je vis Julie [...] ; je la vis, je la reconnus, quoique son visage fût couvert d'un voile. Je fais un cri : je m'élance pour écarter le voile ; je ne pus l'atteindre ; j'étendois les bras, je me tourmentois \& ne touchois rien. Ami, calme-toi, me dit-elle, d'une voix foible. Le voile redoutable me couvre, nulle main ne peut l'écarter. A ce mot, je m'agite \& fais un nouvel effort ; cet effort me réveille ; je me trouve dans mon lit accablé de fatigue, \& trempé de sueur \& de larmes». (Jean-Jacques Rousseau, La Nouvelle Héloïse ou Lettres de deux amants, habitans d'une petite ville au pied des Alpes, Amsterdam, Marc-Michel Rey, 1763, V partie, lettre IX, p. 151, 153-154. Nous reproduisons la graphie et la ponctuation de cette édition.)

6 Journal d'un voyage fait aux Indes orientales, éd. Frédéric Deloffre et Jacques Popin, Paris, Mercure de France, 2002 (abréviation: Journal de voyage; dans les notes: JV21). 
Dans les cinq premières nouvelles, la nuit est donc le cadre d'activités plutôt liées à la sphère de l'intimité, ce qui n'exclut pas qu'elle ait une portée significative liée à son pouvoir de suggestion.

Le soir est le moment où l'on passe en revue les événements de la journée avec les membres de la famille réunis autour d'une table. C'est le soir qu'on s'épanche. C'est durant le "souper» que le vieux Dupuis se lance dans une explication d'où il ressort qu'un père doit toujours rester maître de son bien et garder son autorité sur ses enfants. Mais ce qu'il confie là ne l'empêche pas d'éprouver beaucoup de sympathie pour son futur gendre, Des Ronais, et d'affection pour sa fille Manon ; il ne le dit, dans un esprit de conciliation, que pour éclairer sa conduite qui pourrait paraître trop sévère.

$C^{\prime}$ est le soir aussi qu'a lieu la terrible explosion de colère du père de Des Prez qui croit comprendre que son fils souhaite entrer en religion. Le père a attendu le retour de son fils pour s'expliquer avec lui et lui donner son sentiment. Il n'a pas «soupé» expressément pour être sûr de le voir et de lui donner son sentiment sur la vocation monastique : «En rentrant le soir dans le logis, j'appris qu'il était dans une terrible colère contre moi, et qu'il avait déjà deux ou trois fois demandé si je n'étais pas revenu, et n'avait pas voulu souper sans moi». ${ }^{8}$ L'altercation ne permet pas au jeune homme de se justifier. Elle fournit seulement à la rage du père l'occasion d'exploser dans une crise particulièrement théâtrale. On imagine l'emportement du personnage, son débit haché, on croit voir la gesticulation qui accompagne l'énoncé du discours :

Il s'était déjà emporté contre le couvent, ce fut bien pis quand il me vit. Parbleu, Monsieur, me dit-il, vous me préparez une belle récompense! Avez-vous peur de n'avoir pas de quoi vivre, ou de n'en pas gagner, que vous voulez jurer d'en gueuser? Si je vous croyais, ajouta-t-il avec une fureur terrible, l'âme assez basse pour vous jeter dans un couvent, je vous torderais (sic) morbieu le cou tout à l'heure; ou je vous enfermerais dans un endroit où vous seriez aussi bien claquemuré pour le moins. Fi au diable, poursuivit-il, misérable âme de boue, et de crapule! Vous n'en serez morbieu pas pendant ma vie, c'est de quoi je vous réponds ; j'y mettrai bon ordre.

7 IF, p. 50-52.

8 IF, p. 257.

9 IF, p. 267. 
Le même homme, ici violent et emporté, constatant plus tard que sa volonté n'a pas été respectée, décidera froidement, durant la nuit, de mettre en place un piège destiné à détruire le couple qui a osé le défier. La colère froide débouche non pas sur l'esprit de conciliation dont témoignait le vieux Dupuis, mais sur une volonté de destruction qui conduira à une mort tragique l'épouse de son fils, Madeleine de l'Epine.

Certaines nuits sont éprouvantes : on ne peut y trouver le repos, précisément parce qu'on se remémore dans la solitude les problèmes qui ont surgi et sont restés sans solution. Le calme nocturne leur donne un relief accru, qui empêche le sommeil mais permet de prendre des résolutions, après de longs débats intérieurs dont les éléments s'affrontent dans l'esprit de l'insomniaque. C'est la nuit, à l'écart de l'agitation du jour, qu'Angélique, la mère de Contamine, la mère de Des Frans et le père de Des Prez, que nous venons d'évoquer, prennent leurs décisions, avant de s'endormir enfin, sûrs de leurs choix. Angélique, ne pouvant oublier sa rencontre humiliante avec la princesse de Cologny à la Foire Saint-Germain et l'épisode des miroirs, réfléchit longuement pendant la nuit à ce qu'elle doit faire pour préserver sa réputation : «Elle se coucha sitôt que Contamine fut parti, et rêva ${ }^{10}$ à ce qu'elle avait à faire. Elle était résolue de se déclarer ; mais les moyens lui en paraissaient difficiles. Elle craignait qu'on ne la fît pas parler à la princesse, si elle y allait elle-même. Elle craignait encore quelque insulte de la part des domestiques, qui pouvaient ne la regarder que comme la princesse l'avait regardée elle-même. Dans ce moment, elle se ressouvint de Mademoiselle Dupuis, et résolut de la prier de lui rendre service». ${ }^{11}$ La mère de Contamine et Contamine lui-même délibèrent eux aussi de nuit : «elle [Madame de Contamine] le consola elle-même, et l'envoya reposer. Elle se coucha dans l'incertitude de ce qu'elle avait à faire, mais avant qu'elle s'endormît, elle prit sa résolution [...]. On lui avait rapporté, qu'il [Monsieur de Contamine] avait très mal passé la nuit ; qu'il n'avait fait que soupirer». ${ }^{12}$

Les activités sociales, les "commerces» comme dit Challe, se déroulent souvent de nuit. Le jeu constitue l'une de ces activités vespérales qui peuvent se prolonger très tard. Les romans et les tableaux du temps confirment qu'il s'agit d'une activité courante, tant chez les jeunes gens

10 Le mot «rêver» n'entretient à l'époque aucune relation avec l'univers des rêves, il signifie simplement «songer», «réfléchir».

11 IF, p. 119.

12 IF, p. 133. 
que chez les adultes. Au début du XVII ${ }^{\mathrm{e}}$ siècle, Le Caravage, Georges de La Tour et leurs épigones ont laissé plusieurs tableaux présentant, dans une atmosphère nocturne, des joueurs assis autour d'une table. Des Prez se débrouille pour nouer avec Madeleine de L'Epine un commerce qui paraît innocent, et qui l'est effectivement à ce moment-là. Le jeu leur sert d'alibi. «Lorsque le temps ne permit plus d'aller le soir à la promenade, j'allai jouer chez elle. Nous y jouions très petit jeu, et seulement pour le commerce, et pour avoir prétexte d'y aller tous les soirs [...]. Nous jouâmes donc tous les soirs [...]. Nous ouvrîmes notre trésor à la Saint-Martin, ${ }^{13}$ et quoique nous eussions joué fort petit jeu, il ne laissa pas d'être assez riche pour nous divertir parfaitement bien [...] ; on passa une soirée la plus agréable que j'aie passé[e] de ma vie». ${ }^{14}$ Dans l'histoire de Des Prez, le jeu débouche sur d'autres manifestations liées au calendrier des fêtes religieuses. On fête deux ou trois fois les Rois. ${ }^{15}$ On assiste bien sûr à la messe de Minuit, ${ }^{16}$ avant de participer à un grand bal.

Mais c'est la nuit même de la mort de sa femme que le vieux Dupuis se rend chez le marquis de Verry pour y jouer :

Elle mourut, comme je vous ai dit, il y a environ quatre ans et demi, aux jours gras ; le propre jour de sa mort son mari masqua et alla chez le marquis de Verry. Ce marquis donnait à souper, après lequel il devait y avoir bal [...]. Il avait été averti de la mort de Mademoiselle Dupuis [= l'épouse de Dupuis] [...]. Dupuis fort proprement masqué entra dans la salle, [... il] gagna six cents louis ; et c'était, à ce qu'il disait, la seule journée de bonheur qu'il eût eu en sa vie, mettant la mort de sa femme et son gain dans le même rang.

Comme il avait joué beau jeu, on le prit pour un homme très riche, du moins ses manières le disaient. On le pria de se démasquer, il parut vouloir s'en défendre d'abord ; mais enfin il se démasqua. Le marquis qui le reconnut fit un grand cri. $^{17}$

Ce qui frappe ici, c'est le contraste entre le deuil et les jours gras, le bal masqué et le jeu, qui permet au vieux Dupuis de manifester son

La Saint-Martin se fête le 11 novembre.

14 IF, p. 227.

15 Id.

16 «Nous fîmes la messe de Minuit»: IF, p. 232.

$17 I F$, p. 22-24. 
ressentiment. De plus, l'ambiance nocturne renforce l'aspect théâtral de la scène : la situation et l'éclairage focalisent brutalement l'attention sur un protagoniste, entraînant d'abord une réaction de surprise puis l'hilarité : «Je vous donne à penser dans quels sentiments il laissa ses auditeurs qui s'éclatèrent de rire. Le marquis le traita de fou et de brutal». ${ }^{18}$

D'autres «commerces» nocturnes sont possibles. On peut chanter : le cousin de Babet Fenouil «lui dit un jour, qu'il avait un ami qui chantait autant bien qu'homme du monde [...], j'y allai dès le soir même. [...] L'opéra était tous les jours au logis». ${ }^{19}$ Et voilà nouée la relation entre Babet et Jussy.

On peut aussi se raconter des histoires pendant ou après le «souper». ${ }^{20}$ Les laquais congédiés après qu'ils ont desservi, on se retrouve entre soi et la soirée peut se prolonger fort tard. Ce moment nocturne constitue visiblement un des temps forts de la vie sociale. On en a la preuve dans le récit cadre, puisque la plupart des nouvelles enchâssées sont rapportées de nuit. A la fin de l'histoire de Jussy et de Babet Fenouil, toute la compagnie se rend au chevet de Madame Dupuis pour «souper» auprès de son lit et écouter Dupuis le libertin raconter l'histoire de Des Prez et de Madeleine de l'Epine. Et c'est quand Dupuis le libertin a fini de raconter cette histoire que Contamine surgit comme un diable hors d'une boite : il vient rechercher son épouse, qui devrait être au logis à cette heure tardive. Dans un premier temps, personne ne reconnaît la voix de celui qui confirme une remarque de Madame de Contamine et semble défier par son intrusion tout le groupe des amis rassemblés. Il faut qu'il sorte de l'ombre pour que les autres convives l'identifient et l'interpellent. Comme dans certains tableaux des frères Le Nain, on croit voir surgir de la pénombre tout un groupe suspendu aux lèvres d'un conteur - et même, un des personnages semble nous regarder :

Madame de Contamine, [ne put s'] empêcher de dire, que presque toutes ces sortes de mariages faits à l'insu ou malgré les parents, ne sont jamais heureux. Monsieur Des Prez en est une preuve vivante par sa douleur éternelle; si ce que Monsieur Dupuis vient de dire est vrai. - Il l'est, j'en suis garant, dit une voix inconnue. Chacun jeta les yeux du côté qu'elle venait, et ... - Cela est beau, [...]

18 IF, p. 24.

19 IF, p. 188-189.

20 Les récits ne se font pas obligatoirement de nuit. Des Frans et Dupuis le libertin entament chacun la relation de leur propre histoire après le repas de midi, mais les récits, fort longs, se prolongent dans la nuit (IF, p. 298 et 438). 
de retenir des femmes mariées [...] et d'obliger leurs maris de venir les chercher à minuit ? $?^{21}$

Les mariages font partie des rites sociaux. Deux mariages clandestins, deux mariages secrets, se célèbrent de nuit. ${ }^{22}$ Le premier, le plus théâtral, celui de Jussy et de Babet Fenouil, se fait la nuit même de l'arrivée de Des Frans à Paris, ${ }^{23}$ après des démarches officielles effectuées très rapidement auprès l'archevêque de Paris «afin qu'ils [Jussy et Babet Fenouil] pussent être épousés à minuit avec les solennités ordinaires. [...] Minuit sonna, nous allâmes tous à l'église, le mariage y fut célébré et l'enfant légitimé [...] Nous rentrâmes à Paris sur les quatre heures du matin». ${ }^{24}$ Jussy, que tout le monde croyait mort au Portugal, est donc finalement parvenu à épouser Babet en dépit de l'opposition de l'oncle de celle-ci, Monsieur d'Ivonne, et du ban de six ans auquel ce dernier avait réussi à faire condamner le jeune homme. Les jeunes mariés décident d'organiser le lendemain soir une confrontation spectaculaire avec les tuteurs abusifs de Babet. Il faut que le triomphe des jeunes gens soit public et que la déroute des autorités familiales soit l'occasion d'une grande fête. Pour que celle-ci soit joyeuse et la vengeance complète, Jussy a "envoyé quérir ses deux frères et deux de ses amis tandis que Babet a envoyé quérir quelques-unes de ses bonnes amies». La rencontre tourne à la débâcle pour Monsieur et Madame d'Ivonne. Jussy réussit une entrée remarquée. D'abord, il se fait longuement attendre. Le silence s'installe. Son apparition crée la stupéfaction puisque les $d^{\prime}$ Ivonne se trouvent en face $d^{\prime}$ 'un homme qu'ils croyaient mort : "Jussy entra précédé d'un laquais qui portait un flambeau. Il n'avait point de chapeau, et était comme peut être chez lui le maître de la maison ; mais vêtu d'un air qui me surprit moi-même». ${ }^{25}$ Furieux, les d'Ivonne quittent le terrain; seul reste leur fils. "Nous soupâmes fort bien et avec joie, on chanta ; et comme la compagnie était assez nombreuse, on envoya chercher des violons, on dansa, et il se fit une manière de bal qui

21 IF, p. 289.

22 C'est tout l'inverse des mariages qui se célèbrent de jour avec un certain luxe, comme celui de Des Prez et de Madeleine de l'Epine, ou avec un sens aigu de la provocation, comme celui de Terny et de Clémence de Bernay.

23 IF, p. 13.

$24 I F$, p. 213

25 IF, p. 215-216. 
n'a fini que mercredi matin, avant-hier à trois heures». ${ }^{26}$ Tout comme dans l'épisode au cours duquel le vieux Dupuis gagne au jeu, l'obscurité renforce la dimension spectaculaire de l'épisode.

Le second mariage clandestin, celui de Des Frans et de Silvie, n'a pas grand-chose à voir avec cette explosion de joie vengeresse. La cérémonie est célébrée presque à la sauvette : "A minuit nous allâmes à SaintPaul $^{27}$ qui n'était qu'à deux pas de là. Nous y fûmes mariés et nous rentrâmes au logis sur les deux heures»". ${ }^{28} \mathrm{Il}$ y a bien des témoins, mais la joie ne semble pas au rendez-vous. La fête est sommaire et le repas expédié discrètement, même si l'on mange "fort bien». Des Frans ne peut pas en dire davantage, puisque, à la différence du mariage précédent, il n'y a pas de festivités le lendemain, pas de réunion des proches, pas de bal. Il n'y a rien à montrer et aucun des amis de Des Frans n'est présent. Mauvais signe, mauvais présage, annonciateur de la catastrophe finale : en effet, c'est la clandestinité de ce mariage qui permettra à Gallouin de croire que Silvie n'était que la maîtresse de Des Frans, et d'imaginer qu'il pourrait la conquérir sans commettre un crime.

Comme la réputation d'une femme demande le secret, certaines rencontres se font de préférence de nuit. Attentif au changement des saisons, Challe, qui a observé que les soirées d'été peuvent se prolonger tard, signale que Des Frans n'entre dans Paris pour retrouver Silvie "qu'à huit heures du soir au mois d'octobre, c'est-à-dire de nuit, afin que personne ne [le] vît». ${ }^{29}$ Les relations de Contamine et d'Angélique se nouent, elles aussi, dans une clandestinité totale. Au début, Angélique ne veut le recevoir que de nuit, par peur du scandale :

[Contamine] lui demanda la permission de venir la voir tous les jours, elle y consentit avec peine; mais à condition que pour que ses visites ne fussent point sues, crainte du scandale, il ne viendrait que le soir, si tard que tout le monde serait retiré ; et que surtout son carrosse ni ses gens n'approcheraient point $[\ldots]$. Vous voulez que je prenne une garde $[\ldots]$ mais afin qu'elle ne trouve pas à redire sur vos visites de nuit, il est à propos que vous passiez pour mon cousin

26 Idem. Les invités ne se sont pas ennuyés puisque le bal s'est prolongé jusqu'au petit matin.

27 L'église Saint-Paul, bâtiment «vaste, massif et obscur», desservait la paroisse la plus peuplée de Paris, aux dires de Challe, dans ses Mémoires (éd. Frédéric Deloffre, avec la collaboration de Jacques Popin, Droz, Genève, coll. «Textes littéraires français», 1996, p. 50). Silvie habite près de là, rue Saint-Antoine.

28 IF, p. 385.

29 IF, p. 383. 
[...] Nous lui dirons même, que ne dépendant pas de vous pendant la journée, vous venez quand vous pouvez. [...] Comme il n'y allait que fort tard, il ne fut jamais ni vu ni connu de personne. ${ }^{30}$

C'est la nuit qu'on fait la débauche. Plusieurs moments de la vie de Dupuis le libertin baignent ou semblent baigner dans une lumière nocturne. Le jeudi gras où, habillé en diable, il circule dans Paris ne s'achève qu'au petit matin, et tout l'épisode de l'oublieux suggère, même si rien ne l'indique précisément, une ambiance nocturne. ${ }^{31} \mathrm{D}^{\prime}$ autres instantanés de la vie quotidienne nous montrent des scènes du Paris nocturne sous un aspect très vivant : c'est le cas de l'anecdote qui réunit, dans la même bouffonnerie, la sage-femme aidant une jeune femme à accoucher et une bande de libertins prolongeant leur beuverie. ${ }^{32}$ L'évocation des Rois chez Madame de Londé est l'occasion d'un tableau de nuit réjouissant dont la toile bien connue de Jordaens, Le roi boit, pourrait sans peine servir $d^{\prime}$ illustration - si elle ne présentait une scène diurne. ${ }^{33}$

Normalement, c'est de nuit qu'ont lieu les activités sexuelles. ${ }^{34}$ La Mousson, la femme de chambre de Madame de Londé, dit qu'«on ne se marie que pour être deux, et travailler à faire un troisième», ${ }^{35}$ mais il n'est pas évident que tous les héros challiens travaillent toujours à faire ce troisième. En revanche le plaisir est souvent revendiqué pour lui-même, indépendamment du désir d'avoir des enfants. Ce désir est d'ailleurs absent de la pensée de Challe. À preuve : la façon dont la grossesse survient laisse souvent les père et mère déconcertés. Un enfant, sauf pour Madame de Contamine qui, visiblement conformiste, incarne ici toutes les valeurs de son milieu, est une gêne dont on se débarrasse en l'abandonnant sans scrupule à la porte des églises pour qu'il y soit découvert au petit matin, ou que l'on place dans un couvent où il est recueilli, avec ou sans signe permettant, au besoin, de l'identifier par la suite. L'enfant qui va être baptisé par Silvie et Des Frans a été trouvé

$30 \quad I F$, p. 102.

31 IF, p. 452-455.

32 IF, p. 481.

33 IF, p. 577.

34 A l'exception bien sûr de la scène des seigles (IF, p. 265-266), de la scène de la Maltaise (IF, p. 442-444), ou encore de la scène d'amour avec la veuve (IF, p. 529-530), trois épisodes dont l'expérience diurne semble accroître le pouvoir de suggestion par le non-respect de l'interdit.

35 IF, p. 566. 
la nuit même. ${ }^{36}$ Ce détail constitue à la fois un mauvais présage, et un rappel de la situation de Silvie, enfant trouvée, que ses parents ont dû abandonner malgré la promesse de mariage qu'ils avaient signée. C'est là le destin de Silvie qui culpabilise et accepte d'endosser la faute de ses parents. D'ailleurs l'expression qui désigne la jeune accouchée, «une fille qui rendait avec douleur le fruit de ce qu'elle avait reçu avec plaisir neuf mois auparavant», ${ }^{37}$ permet de comprendre quelle est l'importance relative du plaisir et du désir d'avoir des enfants. Dupuis explique à sa veuve qu'il a le secret de faire l'amour sans conséquences ; la veuve, sceptique mais intéressée par le sujet, lui demande s'il a vraiment ce secretlà. ${ }^{38}$ Le vieux Dupuis évoquera amèrement les «quatre nuits de plaisir, que nous passâmes à la dérobée». ${ }^{39}$ Quant à Bernay, devenu «amoureux $\mathrm{d}^{\prime}$ une très belle femme $[\ldots]$, il voulait persuader [son ami Terny] que le seul plaisir de la vie était d'avoir une maîtresse, et d'en être aimé». ${ }^{40}$ Des Prez exprime le même sentiment : "Je ne crois pas qu'il y ait au monde un plaisir plus grand que celui d'un pareil commerce [l'amour]. Nous le goûtâmes six mois sans troubles ; sans crainte que d'être surpris lorsque nous passions les nuits ensemble, ce qui arrivait assez souvent ; et ce sont les seuls moments heureux que j'ai passés dans la vie». ${ }^{41}$ Des Prez, encore lui, se réjouit de passer quelques nuits avec Madeleine de L'Epine, après son mariage clandestin, alors qu'elle est enceinte : «je couchai avec elle pour la première fois. J'y passai encore quelques autres nuits pendant quatre mois qu'elle y resta ; mais rarement, crainte de donner matière à soupçon». ${ }^{42}$ Challe trouve aisément, pour exprimer les plaisirs de l'amour, des termes qui traduisent l'emportement de la passion. Rapportant les paroles de Gallouin, Dupuis enregistre : «Silvie [...] m'attendait avec une ardeur inconcevable [...], elle me pressa de nous mettre entre deux draps. Elle se coucha la première, je ne la fis pas attendre longtemps, et l'épuisement où nous nous mîmes l'un et l'autre, nous causa une faiblesse qui nous assoupit». ${ }^{43}$ 
$C^{\prime}$ est encore de nuit que la violence sexuelle est mise en œuvre. C'est ainsi que Valeran, préfigurant Gallouin, essaie de violer Silvie en profitant de son sommeil : «ll est certain que je me réveillai en sursaut à la fraîcheur de sa main qu'il me porta sur l'estomac. Je me mis à crier au secours ; il me saisit au corps ; il fit ses efforts pour me faire taire, et me fit même des violences dont je portai des marques assez longtemps». ${ }^{44}$

C'est de nuit que Madame de Londé surprend son mari en galante compagnie avec la petite paysanne. ${ }^{45} \mathrm{Challe}$ fait preuve en l'occurrence, une fois de plus, d'une remarquable sensibilité aux sensations acoustiques, lorsqu'il observe que pour ne pas alerter le mari adultère, la femme trompée qui veut surprendre son époux sur le fait «mit pied à terre à cent pas du logis, afin que le bruit de son carrosse ne fût point entendu» ${ }^{46}$ dans le silence nocturne. Alors que la scène observée est quasiment identique à celle que vit Des Frans lorsqu'il découvre l'infidélité de Silvie, la réaction de Madame de Londé n'a rien de commun avec le désespoir tragique de Des Frans. Nous restons, avec elle, dans le domaine de la comédie. Dans la présentation plaisante de la scène qui repose sur un coup de théâtre digne des boulevards, il n'y a place pour aucun reproche. La confrontation des deux passages met en évidence une réalité sociologique : la société - ou Challe - distribue des rôles bien différents aux hommes et aux femmes. Un homme n'est en aucun cas tenu de se soumettre à la fidélité absolue qu'il exige de son épouse. L'adultère féminin déclenche le désespoir du mari trompé, tandis que l'adultère masculin ne suscite que le rire de l'épouse surprenant son mari sur le fait : «Elle entra aussitôt dans ma chambre et vint me trouver à mon lit; je n'avais garde de songer à elle. Je savais bien que je vous prendrais sur le fait, me dit-elle en me baisant; mais je vous le pardonne ; car elle est bien jolie, et bien jeune. Après cela elle s'en retourna sans attendre ma réponse». ${ }^{47}$

La façon dont ces épisodes nocturnes sont présentés fait souvent songer à la manière dont Baudelaire, nourri de l'imaginaire du XVIII ${ }^{\mathrm{e}}$ siècle, évoque les choses dans son poème en prose « Les Fenêtres »:

Celui qui regarde du dehors à travers une fenêtre ouverte, ne voit jamais autant de choses que celui qui regarde une fenêtre fermée. Il n'est pas d'objet plus

45 IF, p. 578-579.

46 IF, p. 579.

47 Id. 
profond, plus mystérieux, plus fécond, plus ténébreux, plus éblouissant qu'une fenêtre éclairée d'une chandelle. [...] Dans ce trou noir ou lumineux vit la vie, rêve la vie, souffre la vie. ${ }^{48}$

Baudelaire présente de façon paradoxale le spectacle qu'offre une fenêtre. D'ordinaire, la fenêtre permet d'échapper à l'univers cloisonné de la chambre pour s'évader vers l'extérieur - ce que toute la peinture occidentale confirme depuis Léonard de Vinci, pour qui le cadre de la toile constitue le cadre d'une fenêtre. La clé du paradoxe est fournie lorsque Baudelaire nous fait comprendre que la vision qu'il estime bien plus passionnante, "fenêtres fermées», est une vision nocturne. Ce qu'on peut voir au soleil, ce qui est accessible à tous, est rejeté, comme dénué d'intérêt. Inversant la situation de l'observateur, Baudelaire lui permet d'accéder à une réalité plus intime. De nuit, la fenêtre permet de contempler l'intérieur de la chambre et d'observer ceux qui y sont éclairés par la lumière, tandis que le spectateur, plongé dans l'obscurité, évacue de sa conscience tout ce qui ne dépend pas de ses yeux. Le regard séduit du contemplateur s'apparente à celui du voyeur. L'observateur assiste au spectacle comme l'amateur de théâtre qui regarde, fasciné, les scènes qu'éclairent vivement les feux de la rampe. Mais il est en même temps le démiurge qui crée la magie du spectacle, tout en restant hors-champ. Un exemple supplémentaire confirme cet aspect théâtral des scènes présentées dans Les Illustres Françaises. Dupuis envoie son laquais observer la nuit de noces de Célénie et d'Alaix ; ce laquais explique avec force détails comment il a fait pour être aux premières loges, "au hasard de se casser le cou», et rapporte la scène à laquelle il a assisté avant de conclure : «Je suis venu tout aussitôt vous en faire le récit, bien fâché que vous n'ayez pas été vous-même à la comédie». Quelques notations méritent d'être soulignées :

Mon laquais revint sur les trois heures après minuit, qui me dit qu'il y avait eu un grand souper chez la mère de Célénie [...], qu'après le souper ils avaient été à l'église où ils avaient été épousés. Qu'au retour ils avaient pris le chemin de la maison d'Alaix [...] : qu'il avait fait en sorte de se cacher sur une petite fenêtre qui donnait derrière la ruelle $e^{49} \mathrm{du}$ lit nuptial [...]. Ce laquais qui avait de la bonne foi, m'avoua qu'il y avait eu autant de curiosité dans son fait, que d'envie de me satisfaire [...]. Qu'il avait entendu Célénie entrer dans cette

48 Charles Baudelaire, Petits poèmes en prose, dans CEuvres complètes, éd. M. Jamet, Robert Laffont, coll. "Bouquins ", p. 198.

49 La «ruelle» désigne ici l'espace ménagé entre le lit et le mur. 
chambre [...]. Enfin, me dit mon laquais, je n'entendis plus de bruit du tout, [...] mais après cela j'ai entendu beau jeu [si bien] que de peur d'être surpris en éclatant de rire, je me suis jeté de la fenêtre sur le pavé $[\ldots] .^{50}$

Challe, observateur attentif des spectacles de la nuit, nous les restitue avec un sens du détail qui crée véritablement l'illusion du réel.

Dans les sixième et septième histoires, ce prodigieux metteur en scène exploite de manière magistrale l'obscurité dans laquelle il plonge ses personnages. La nuit n'est plus un simple décor, elle devient un révélateur des passions qui les habitent. On n'oublie pas la nuit obscure où Des Frans reçoit, de la main d'un inconnu, les documents qui sont destinés à lui apprendre le passé trouble de Silvie et les manœuvres auxquelles elle a eu recours afin de s'assurer une ascendance présentable. C'est par une nuit sans lune qu'on lui transmet des nouvelles de nature à le précipiter dans le désespoir le plus absolu. Le lecteur observe ici un de ces effets de notation poétique qui visent à suggérer une correspondance entre une situation atmosphérique et un climat psychologique. Un détail observé avec justesse suffit pour suggérer une ambiance en accord avec la tonalité de la scène :

Je sortais un soir fort tard de chez elle dans le mois de janvier. Il était près de minuit. Le temps était extrêmement sombre ; on ne voyait ni ciel ni terre. Un flambeau qu'un laquais me portait s'était éteint par le vent, et aucune lanterne n'était restée allumée ; je marchais à tâtons. J'entendis quelqu'un auprès de moi $[\ldots]$ - Tenez, Monsieur, me dit-il, on m'a chargé de vous rendre en main propre ce paquet-ci. [...] en me disant cela il me donna un paquet cacheté $[\ldots]$. Je décachetai l'enveloppe dans le moment, comme si j'avais pu lire dans un lieu où je ne pouvais discerner les rues. Je m'aperçus dans le moment de ma ridicule curiosité. $^{51}$

Par la suite, Des Frans déchiffre le message : «Je vous laisse à penser ce que je devins à cette lecture [...]. Je rêvai toute la nuit à ce que je ferais. Je lus et relus cette lettre». ${ }^{52}$ Il se laisse tenter par l'indication que lui fournit la lettre anonyme, se rend à l'auberge indiquée dans le mes-

50 IF, p. 490-491.

51 IF, p. 319; nous soulignons.

52 Id. 
sage et y rencontre l'un des acteurs de la comédie chargé de le berner, Rouvière. Après un repas de midi largement arrosé, ce dernier raconte à Des Frans, sans savoir qu'il s'adresse au protagoniste de l'histoire, l'aventure de ce benêt - Des Frans lui-même - qui doit épouser une jeune aventurière et, enfilant un verre après l'autre, Rouvière et son interlocuteur prolongent la conversation jusqu'en début de soirée. Révolté par ce qu'il vient d'apprendre, Des Frans se rend en pleine nuit chez Silvie pour l'accabler d'injures. L'ivresse, la rage et l'obscurité de la nuit le submergent :

Il était fort tard lorsque nous nous quittâmes. Il gelait à tout briser : la nuit était calme et belle, ${ }^{53}$ un garçon du cabaret me portait un flambeau, mon valet ne m'ayant pas retrouvé $[\ldots]$. Une pointe de vin que j'avais, me présenta une comédie en allant voir ma perfide ${ }^{54}[\ldots]$, j'étais si occupé du régal que je croyais m'aller donner que je ne songeai pas de dire à celui qui me portait un flambeau, de m'attendre [...], lorsque je fus entré chez mes traîtresses. Il était si tard qu'elles allaient se mettre au lit [...]. Elles étaient toutes deux dans un état plus facile à s'imaginer qu'à représenter [...]. Elles gardaient un profond silence, et leur confusion était incompréhensible. Notre scène, quoique muette, était divertissante. ${ }^{55}$

On n'oublie pas davantage la vision troublante de Silvie qui désarme Des Frans pourtant rempli d'une fureur vengeresse :

Je jetai les yeux sur elle dans ce moment; je me perdis. Elle était encore à mes pieds, mais dans un état à désarmer la cruauté même. Elle était toute en pleurs : le sein qu'elle avait découvert, et que je voyais par l'ouverture d'une simple robe de chambre; ses cheveux qu'elle avait détachés pour se coiffer de nuit, et qui n'étant point rattachés tombaient tout le long de son corps, et la couvraient toute ; sa beauté naturelle que cet état humilié rendait plus touchante ; enfin mon étoile qui m'entraînait, ne me firent plus voir que l'objet de mon amour et l'idole de mon cœur. Le puis-je dire sans impiétée ${ }^{56}$ Elle me parut une seconde Madeleine ; j'en fus attendri, je la relevai, je lui laissai dire tout ce qu'elle voulut. Je ne lui prêtai

53 Challe renforce les notations d'atmosphère précédentes; voir note 52 , soit $I F$, p. 319 ; voir aussi $I F$, p. 42.

54 La formule, tout comme la dernière phrase de notre citation, renforce l'aspect théâtral de la scène. Le tour est très elliptique : «me présenta l'idée de jouer une scène de comédie en allant voir ma perfide».

55 IF, p. 333-334; nous soulignons.

56 Pourquoi sans impiété? Parce qu'il n'est pas le Christ bien sûr alors que, si Silvie est Madeleine, il devrait l'être. 
aucune attention ; je n'étais plus à moi. ${ }^{57} \mathrm{~J}^{\prime}$ étais déchiré par mille pensées qui se formaient l'une après l'autre dans mon esprit, et qui se détruisaient mutuellement ; ou plutôt j'étais dans un état d'insensibilité, qui tout vivant que j'étais, ne me laissait pas plus de connaissance qu'à un homme mort. ${ }^{58}$

L'ambiance nocturne accentue le caractère visuel de cette scène au cours de laquelle Silvie tente maladroitement de s'expliquer. Mais ce qui la justifie, aux yeux de Des Frans, ce sont des éléments non verbaux, étrangers au débat: ses pleurs, ses larmes. Des Frans est troublé par le déshabillé tout à fait galant de Silvie. La sensualité s'impose comme un argument décisif lors d'une fulgurante apparition où le corps se laisse deviner dans un espace intermédiaire : le vêtement sert à la fois à révéler et à occulter. Le cadrage d'une image immobile met en évidence, dans le visage, dans les cheveux dénoués ou dans le sein découvert, une esthétique théâtrale. Vêtue d'une simple robe de chambre et les cheveux défaits, Silvie n'est pas «sous les armes»; elle ne l'attendait pas et sa séduction naturelle est aux antipodes de la manœuvre concertée de la veuve qui avait feint un sommeil de jour pour séduire Dupuis. ${ }^{59}$ L'absence de parure, l'émotion due à la révélation d'une séduction sans apprêts liée à un état humilié..., la référence est certainement littéraire, mais elle se trouve ici ravivée, parce qu'elle renvoie à la fois à la vie quotidienne et à un lieu commun pictural. La présentation fait songer aux variations sur le thème de la Madeleine repentante, ${ }^{60}$ thème iconographique que Des Frans évoque d'ailleurs lui-même. La sensualité et la nuit, et la séduction née de la nuit, signent la perte de toute rationalité. Silvie est belle et désirable. La nuit, les repères s'estompent - à la fois physiquement et mentalement - , et Des Frans se laisse emporter par ses sens.

57 Cette suite de phrases hachées, ce ton haletant sont exceptionnels sous la plume de Challe et traduisent une intense émotion. Des Frans est privé de ses moyens ; il est comme mort. La contemplation produit l'extase, «ekstasis». Il est hors de luimême, au sens étymologique.

58 IF, p. 335.

59 IF, p. 529.

60 Cette scène de «la Madeleine repentante» a inspiré entre autres Diderot dans le dénouement de l'histoire de Madame de la Pommeraye et de Monsieur des Arcis. Voir Henri Coulet, "Le thème de la Madeleine repentie chez Robert Challe, Prévost et Diderot», Saggi e ricerche di letteratura francese, Rome, Bulzoni Editore, 1975, n¹4, p. 287-304. 
Dans la descente aux enfers que vit Des Frans, un autre épisode va se graver avec la même intensité dans la mémoire du lecteur : c'est la découverte de l'adultère de Silvie. Il ne s'agit plus cette fois d'un récit qui peut être contesté par la jeune femme. Des Frans voit de ses yeux l'adultère, et touche de ses mains le collier maléfique : «J'avais [...] une clef du jardin de la maison. Je n'avais mandé mon retour à personne ; on ne $\mathrm{m}^{\prime}$ attendait pas. Je voulus arriver à une heure que tout le monde fût endormi ; ce fut à deux après minuit [...] Je montai dans l'appartement de Silvie le plus doucement que je pus pour la surprendre dans son sommeil ; mais je fus bien plus surpris moi-même, lorsqu'à la lumière d'une bougie qui était allumée, je vis les habits d'un homme sur un fauteuil à côté $d u$ lit, et deux personnes couchées ensemble». ${ }^{61}$ Dans le monde romanesque de Challe, l'espace nocturne n'est jamais habité par les rêves. Il n'y a aucune place pour l'onirisme. Lorsque Des Frans découvre Silvie dans les bras de Gallouin, c'est bien un cauchemar qu'il vit, mais pas un instant il ne songe à se demander si ses sens le trompent, s'il est l'objet d'une vision mensongère. Il ne lui est pas nécessaire de toucher pour confirmer le témoignage de ses yeux.

La scène, éminemment plastique, appelait l'illustration; et de fait, dès la première édition, celle de 1713 , c'est ce motif qu'illustre la gravure de Coster (voir Figure 1). Or, que découvre-t-on dans cette gravure? Ce n'est pas le monde de Coypel, de Watteau ou de Pierre Yver, auquel par les hasards de l'édition Les Illustres Françaises se trouvent associées ; c'est le monde de La Tour ou de Le Nain, voire du Caravage, un monde trouble où l'ombre le dispute à la lumière de la chandelle, où la sensualité se mêle aux pulsions meurtrières. La gravure présente en un raccourci synthétique, plus synthétique que ne l'est la narration de Des Frans, l'épisode le plus dramatique de la sixième histoire. Comme un projecteur de théâtre disposé sur la droite, un candélabre éclaire la scène ; une large flaque d'ombre s'étale sur le sol, au pied du cierge. La lumière éclaire simultanément Des Frans et le couple adultère plongé dans un sommeil réparateur. La main droite de Gallouin s'égare encore en un geste sensuel sur le sein de Silvie. Au centre de cette composition, Des Frans se dresse en habit de voyage (chapeau, bottes, fouet de postillon à la ceinture). Il se tourne vers les spectateurs, c'est-à-dire vers nous, les lecteurs, et tient dans sa main droite le fatal collier qui, après avoir été l'instrument du charme qui a provoqué «l'adultère innocent», doit lui servir à prouver la culpabilité de Silvie en cas de dénégation de sa part. Dans le fond, on devine Madame Morin plongée dans un som-

61 IF, p. 409 ; nous soulignons. Sur le sujet du rêve, voir ci-dessus, note 5. 
meil dont on imagine qu'elle ne reviendra pas: "Quelle vue! Quelle rage! Quel désespoir! Imaginez-vous ce que je devins. Je mis l'épée à la main dans le dessein de les percer l'un et l'autre ; mais un mouvement qu'elle fit me désarma. Je jetai les yeux sur ce sein que j'idolâtrais. ${ }^{62}$ Toute ma fureur m'abandonna, je n'écoutai plus ma rage que pour plaindre mon malheur». ${ }^{63}$ Une nouvelle fois, en écartant la rationalité, la sensualité entraîne la suspension du jugement, l'irrésolution.

Dans la littérature classique, une tradition du fantastique nocturne court des Histoires tragiques au Manuscrit trouvé à Saragosse, de François de Rosset à Jan Potocki. ${ }^{64}$ La nuit est le domaine des suspensions de la raison et des manifestations diaboliques, des noctium fantasmata. Bien que Challe utilise souvent le mot «diable» comme interjection, le diable n'apparaît jamais, ne se matérialise jamais dans ses récits, ni de jour ni de nuit. A peine pourrait-on rappeler que des comédiens travestis en diables organisent un spectacle dans la Continuation de Don Quichotte ${ }^{65}$ ou qu'un soir de débauche, un «jeudi gras», dans un «grand bal au faubourg Saint-Germain», Dupuis le libertin se déguise en diable "tout garni de sa queue et de ses griffes» ${ }^{66}$ en revêtant les hardes qui lui sont attribuées par le sort. Il n'y aura que des gens crédules, une foule stupide - Ad populum phaleras ${ }^{67}$ - , pour croire à la réalité d'une présence diabolique. On pourrait cependant observer que, dans Les Illustres Françaises, toutes les mauvaises nouvelles sont apportées à leurs destinataires de nuit. Si le diable n'existe pas physiquement dans l'univers romanesque de Challe, il ne faudrait cependant pas croire que son action soit inexistante. Simplement cette action s'explique ration-

62 C'est à nouveau par la séduction sensuelle qu'exerce Silvie, et non par ses paroles, qu'elle désarme Des Frans. Cette scène rappelle celle de la p. 335.

63 IF, p. 409.

64 François de Rosset, Histoires tragiques, éd. Anne de Vaucher-Gravili, Paris, Le Livre de poche, 1994 : Histoire $X$, «D’un démon qui apparaissait en forme de damoiselle au lieutenant du chevalier du guet de la ville de Lyon. De leur accointance charnelle, et de la fin malheureuse qui en succéda». Voir aussi Jan Potocki, Manuscrit trouvé à Saragosse, éd. René Radrizzani, Paris, Le Livre de poche classique, 1992, dixième journée, « Histoire de Thibaud de la Jacquière », p. 121-129.

65 Robert Challe, Continuation de l'Histoire de l'admirable Don Quichotte de la Manche, éd. Jacques Cormier et Michèle Weil, Genève, Droz, 1996.

66 IF, p. 451-452.

67 «Balivernes bonnes pour le peuple»; l'expression reparaît à plusieurs reprises dans le JV21. 
nellement. Dans les Difficultés sur la religion, ${ }^{68}$ Challe dira : «Il ne faut assurément que la nature et la constitution de notre machine pour nous tenter, voilà le diable ; il ne faut que notre raison pour nous porter à combattre la tentation, voilà la grâce ; il ne faut que la force de notre libre volonté pour vaincre, et voilà tout le secours qui nous est nécessaire, le seul que Dieu donne, ou puisse donner en agissant en dieu». ${ }^{69}$ Pourtant, Des Frans, plus réservé sur le rôle de la volonté que l'auteur des Difficultés, dira que la volonté n'est pas seule à l'œuvre : «Si nos actions sont tout à fait volontaires, du moins peut-on dire, que notre vie n'est pas toujours gouvernée par notre seule volonté, et que l'étoile en règle les principaux mouvements et la disposition». ${ }^{70}$ De fait, la nuit entraîne l'occultation de la volonté : la machine peut agir d'elle-même. Durant la nuit, les forces de la volonté sont prêtes à capituler. C'est la nuit que Silvie cède à Gallouin. Comme le dira Goya dans l'épigraphe d'une de ses gravures, «le sommeil de la raison engendre les monstres».

Dans le cas de Silvie, le retour de la lumière du jour coïncide avec le retour de la raison. En se réveillant dans les bras de Gallouin, elle retrouve ses inhibitions et sa volonté. Gallouin se souvient : "Je me réveillai le premier. Je voulus encore la caresser, je ne trouvai plus cette Silvie toute ardente et toute passionnée, elle se souvenait de tout ce qui lui était arrivé la veille et une partie de la nuit, mais elle le détestait. Je ne vis plus dans elle qu'une furieuse ; elle s'arracha de mes bras, elle appela du monde, et cria au secours à pleine tête». ${ }^{71}$

Étrangement, voulant dans les Difficultés prouver l'existence de deux principes, l'âme et le corps, l'auteur des Illustres Françaises évoque un exemple qui renvoie à la situation centrale de la sixième histoire de son roman : «Qu'une femme d'honneur ait été trompée sous l'apparence de son mari, loin d'avoir senti quelque douleur corporelle, elle aura senti le plus vif des plaisirs ; cependant elle souffrira une peine mortelle en découvrant la fourberie; fût-ce même dans le temps de l'action. Qui peut être la partie souffrante en cette occasion? Ce n'est assurément

68 Robert Challe, Difficultés sur la religion proposées au père Malebranche, éd. Frédéric Deloffre et François Moureau, Genève, Droz, 2000 (abréviation : Difficultés).

69 Difficultés, p. 425. Voir aussi : «Quoique jeune et que je n'eusse qu'environ dix-huit ans, la force de l'éducation est telle que je ne cessai pourtant point d'être dévot, et de me couvrir de signes de croix depuis la tête jusqu'aux pieds dans la crainte excessive où j'étais des diables, des revenants et des sorciers» (Difficultés, p. 85).

$70 I F$, p. 308.

71 IF, p. 547. 
pas le corps». ${ }^{72}$ Cette réflexion permet d'éclairer la relation trouble qui unit Silvie à Gallouin. Au moment même où sa sensualité lui rappelle le plaisir éprouvé par son corps, son âme et sa volonté - occultées par le "charme» du collier - lui font détester celui qui a abusé d'elle en profitant de son sommeil artificiel. ${ }^{73}$

Tout en se rattachant au récit de la Marquise de Ganges tel qu'il a paru dans l'édition des Histoires tragiques de 1685, la suite de la nouvelle préfigure les romans noirs de la fin du XVIII siècle. Très sensible à l'atmosphère inquiétante d'un château en ruines et à la lumière funèbre que diffuse le crépuscule, Challe évoque l'ambiance lugubre de ce château-prison: «Mon valet me l'amena dans la maison où je l'attendais, qui était un reste de la mienne que le feu avait épargné, ${ }^{74}$ et que j'avais fait raccommoder pour servir à mon dessein. Je la fis monter dans une chambre sans qu'elle vît qui que ce fût que moi. Cette chambre avait pour tout meuble un méchant lit de camp, et une paillasse sans linceuls ${ }^{75}$ ni couverture, une selle ${ }^{76}$ de bois à trois pieds comme elles sont en province, sans tapisserie, sans foyer, ni cheminée, ni fenêtre, ne recevant le jour que par un œil-de-bœuf, que j'avais fait laisser en haut, et qui était condamné par une grille de fer. Quoique le soleil fût couché, il y avait assez de jour encore pour discerner les objets». ${ }^{77}$

C'est dans cette chambre dépouillée, voire sordide, que Silvie, humiliée par les soins de Des Frans et éclairée d'une seule chandelle, se transformera en une seconde Madeleine, la Madeleine pénitente, dégradée et émaciée, que présentent d'autres tableaux du XVII ${ }^{\mathrm{e}}$ siècle ${ }^{78}$ : "Je lui jetai un paquet de hardes qui pouvaient servir à la dernière des

72 Difficultés, p. 582.

73 Ce passage illustre et complète à merveille les fines observations de Marc-André Bernier qui examine comment, des Illustres Françaises aux Difficultés sur la religion, Challe essaye de faire la part de la volonté et de la sensualité dans le comportement humain : voir «Entre diablerie et fabrique du corps : les éclipses de l'âme dans l'histoire de Silvie» dans Marie-Laure Girou-Swiderski (textes présentés par, avec la collaboration de Pierre Berthiaume), Challe et/en son temps, Paris, Champion, 2002, p. 39-46. Jacques Popin épingle le même passage des Difficultés pour le rapprocher, comme nous, de l'histoire de Silvie ; voir Poétique des Illustres Françaises, Mont-de-Marsan, éditions Interuniversitaires, 1992, t. I, p. 203-204.

74 Noter encore une fois la valeur symbolique du décor et de l'heure (la fin du jour).

75 Linceuls, au sens ancien de «draps».

76 Etymologiquement, le terme désigne une chaise, soit ici un trépied.

77 IF, p. 414-416.

78 Voir deux tableaux contemporains du XVII ${ }^{\mathrm{e}}$ siècle au musée Fabre de Montpellier. 
paysannes. Je la fis déshabiller, je l'obligeai à se couper elle-même les cheveux que je brûlai en sa présence à une chandelle. ${ }^{79}$ Je les regrette encore : je n'en ai vu de ma vie de plus beaux, ni de plus longs, ni en plus grande quantité. J'emportai tout ce qu'elle avait apporté sur son corps, je l'obligeai de se couvrir des hardes que je venais de lui donner, et ne lui laissai ni bas ni souliers. Ce fut ainsi que je la mis pour le corps, et pour la nourriture, je lui laissai du pain noir et de l'eau, et n'allai plus lui en porter que tous les trois jours».

La septième histoire des Illustres Françaises s'achève sur une ultime scène de nuit : la mort de Gallouin, «branché» avec son compagnon au clair de la lune. Une scène dont la sèche précision graphique - deux silhouettes s'agitant sur la ligne de l'horizon nocturne - rappelle les eaux-fortes impitoyables de Jacques Callot : «[Gallouin] avait été le samedi saint dans un endroit où sa charité et son zèle l'avaient conduit. Il se retirait dans son couvent à deux lieues de là avec un frère [...]. Il fallait nécessairement qu'ils passassent par une forêt [...]. Il y avait dans cette même forêt des voleurs qui avaient fait des désordres et des crimes épouvantables. [...] Ils aperçurent au clair de la lune ces deux pauvres capucins [...]. Ils résolurent de les tuer ; mais de peur que leurs corps n'indiquassent leur crime, ils les pendirent à des arbres». ${ }^{81}$

Autant d'images inoubliables qui, s'associant à des tableaux de l'époque, se gravent dans la mémoire du lecteur.

Concluons pour Les Illustres Françaises. Dans la plupart des nouvelles, la nuit constitue l'espace dans lequel se nouent les divers "commerces de la vie». Les nombreuses notations visuelles s'associent spontanément dans l'esprit du lecteur aux tableaux de La Tour, du Caravage, voire de petits maîtres flamands du XVII ${ }^{\mathrm{e}}$ siècle. Dans l'Histoire de Silvie et de Des Frans, qui couvre la sixième nouvelle et une large part de la septième, l'exploitation des pouvoirs de la nuit est l'occasion d'une réflexion quasi métaphysique : l'occultation de la raison due à l'obscurité permet à l'instinct et à la sensualité de l'emporter sur les forces de la volonté.

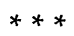

79 Traditionnellement, la femme adultère est tondue ; c'est un signe manifeste de son inconduite. Mais ici, la splendeur des cheveux de Silvie faisait tout le charme de sa personne, d'où l'importance de l'humiliation. Des Frans en la mutilant ressent une souffrance proche du masochisme.

80 IF, p. 416.

81 IF, p. 586. 
Seul romancier de sa génération à enregistrer des précisions visuelles et des notations d'atmosphère qui constituent le décor des scènes qu'il nous donne à voir, Challe nous impose aussi sa vérité, sa vraisemblance, sa «mise en image» dans d'autres œuvres où se retrouvent également des séquences nocturnes inoubliables.

Ainsi en est-il d'un passage des Mémoires, où Challe évoque la cupidité d'un personnage, révélée au cours d'une nuit de tempête, et le châtiment que celui-ci s'imposera au cours d'une nuit d'horreur :

M[onsieur] de Beuvron [...] alla un jour à la chasse du côté de ce château [celui $\mathrm{du}$ frère du président Jean-Jacques de Mesme]. Il suivait un sanglier qu'il avait fait forcer. Une pluie très forte accompagnée d'éclairs et de tonnerre l'obligea de chercher un asile. Il entra dans le château, et comme il y était parfaitement connu, le concierge le fit monter dans une chambre où il y avait du feu. Il y fut reçu fort civilement par une femme d'environ cinquante-cinq ans et par un ecclésiastique. C'était la tante et le cousin du président de Mesme. ${ }^{82}$

Tandis que la nuit tombe et que l'orage gronde, la tante du président de Mesme entreprend de raconter comment son fils et elle ont été spoliés par le président. Le lendemain, le marquis de Beuvron s'empresse de rapporter personnellement cette escroquerie au Roi, à Versailles, afin que justice soit faite et que le tuteur indélicat rende gorge. Le Roi ordonne sur-le-champ la restitution de l'héritage. Touché dans son honneur par la décision royale, le président de Mesme se retire dans son cabinet pour y passer une mauvaise nuit : «On le trouva mort le lendemain samedi, la tête sur le bureau, et les jointures des doigts de ses mains en dehors mangées à belles dents». ${ }^{83}$ Cette étonnante formule est marquée par un relief puissant qui rappelle l'Ugolin du Dante enfermé dans sa cellule. La rage d'avoir été humilié en public et d'avoir dû restituer ce dont il s'était emparé indûment ont amené le président à se ronger les sangs jusqu'à ce que mort s'ensuive.

Dans le Journal de voyage, l'épisode des trois rats voleurs d'œufs sur le navire se déroule de nuit lui aussi, et se présente comme un spectacle de théâtre, de foire ou de cirque. La mise en scène imaginée par Challe nous transporte à fond de cale, "où il y a toujours une lampe allumée», ${ }^{84}$

84 Toujours ce même souci d'expliquer matériellement le pourquoi des choses : Challe a pu observer le manège des rats sans les déranger, parce qu'ils sont habitués à la lumière, toujours présente dans cette partie du bateau. 
«à minuit et demi». Suit une petite saynète dont Challe et cinq hommes d'équipage sont les spectateurs et qui rappelle les vignettes dessinées par François Chauveau pour accompagner les Fables de La Fontaine. Une fois encore, Challe recourt au mode de présentation nocturne évoqué par Baudelaire : les spectateurs sont plongés dans l'obscurité tandis qu'une lumière violente éclaire les acteurs, trois rats qui s'entraident mutuellement, comme des hommes, pour voler trois œufs.

Il y avait très longtemps que notre chirurgien accusait ses garçons de manger les œufs des malades : il avait beau les compter, il s'en trouvait toujours à dire le lendemain deux ou trois, et quelquefois quatre, quoiqu'il eût lui-même la clef du réduit qu'on lui avait fait dans le fond de cale en avant de l'eau, où il y a toujours une lampe allumée [...]. Pour ce sujet, on a percé, avec une vrille de charpentier, à cinq endroits différents, la cloison de ce réduit du chirurgien, et nous sommes descendus dans le fond de cale, à la fin du premier horloge du quart de la nuit, c'est-à-dire à minuit et demi. [...] Nous n'avons fait aucun bruit et avons pris chacun possession de notre trou, au nombre de six spectateurs [...]. Nous ne nous sommes point ennuyés : les voleurs sont venus presque aussitôt que nous.

Trois gros rats, qui sont arrivés en même temps, et qui se sont approchés du baril où étaient les œufs. Ce baril est à demi vide. L'un de ces rats est descendu dedans, un autre s'est mis sur le bord, et l'autre est resté en bas en dehors. Nous n'avons point vu ce que faisait celui qui était dans le baril, les bords en étaient trop hauts ; mais un moment après, celui qui était au haut a paru tirer quelque chose en se retirant de dedans, où il s'était baissé. Celui qui était resté en dehors, en bas du baril, a monté sur les cercles, et, appuyé sur ses pattes de derrière, s'est élevé et a pris dans sa gueule ce quelque chose que celui qui était sur le bord en haut tenait. Celui-ci, après lui avoir lâché prise, a replongé dans le baril et a encore tiré à lui quelque chose qui a été aussi repris par celui qui était sur les cercles en dehors. On a pour lors reconnu que c'était la queue d'un rat ; et à la troisième tirade le rat voleur a paru, tenant entre ses quatre pattes un œuf, le dos appuyé contre le dedans du baril et la tête en bas. ${ }^{85}$

L'anecdote conduit Challe à des considérations sur l'évidente incapacité de l'homme à connaître Dieu, à donner une explication à l'univers, et sur l'impossibilité de connaître nos fins dernières. La conclusion s'impose : de la plante à l'homme, la matière est douée de qualités similaires qui ne trouvent de véritable expression que chez l'homme, mais qui existent à l'état plus ou moins latent dans tous les êtres vivants. La 
pensée est possible indépendamment de la conscience de soi. ${ }^{86}$ Challe achève le développement par une observation qui lui est personnelle, même si elle trouve partiellement sa source chez saint Augustin ${ }^{87}$ : «Malheureux que nous sommes, nous ne nous connaissons pas nous-mêmes, et plus malheureux encore, de ce que nous ne cherchons point à nous connaître». Relevons que, comme d'habitude, Challe réinterprète ses références culturelles en y exprimant son inquiétude métaphysique. La dernière observation est précédée $d^{\prime}$ une remarque qui révèle la permanence de cette inquiétude : «L'homme ne reconnaîtra-t-il jamais son ridicule orgueil, assez vain pour le pousser à vouloir connaître Dieu lui-même ?» Ceci rejoint ce qu'il avait déjà dit précédemment, à la date $\mathrm{du} 1^{\mathrm{er}}$ juillet 1690, quand il essayait de situer la place de l'homme dans l'échelle des êtres: "L'homme ne s'étudie pas soi-même avec assez d'attention, \& entraîné par son ridicule amour-propre, il s'adjuge la prééminence sur toutes les autres espèces et s'en tient servilement à son jugement sans vouloir approfondir que les animaux qu'il nomme brutes ont droit d'en appeler au tribunal de leur commun Créateur». ${ }^{88}$ L'intelligence n'est jamais qu'une qualité parmi d'autres, qui n'autorise pas l'homme à se croire supérieur à toute la création. Les animaux sont à égalité avec l'homme devant la justice de Dieu. Corollaire de la découverte galiléenne, l'homme perd sa place privilégiée : il n'est plus au centre de l'univers.

Challe emprunte sans doute cet épisode au monde des livres. ${ }^{89}$ Mais une autre séquence renvoie plus directement à son expérience personnelle. Challe rapporte une anecdote qui le met lui-même en scène avec son chien. Alors qu'il se croit perdu, de nuit, dans une forêt du Poitou - «La nuit était si obscure que je n'en voyais pas la tête [de son cheval], bien loin de pouvoir distinguer où il mettait le pied ${ }^{90}-$, Challe observe que son chien retrouve, sans hésitation, le chemin du village. Cette expérience le convainc à nouveau que le chien, pas plus que les autres animaux, n'est une simple machine. C'est à une conclusion du

86 Le sujet avait été largement débattu par Malebranche dans La Recherche de la Vérité, livre VI, II, 7, paru en 1675, et bien sûr dans les livres IX et X des Fables de La Fontaine (1678-79).

87 «Nul homme ne sait ce qui se passe dans l'homme, sauf l'esprit de l'homme qui est en lui» (Saint Augustin, Confessions, X, 5, cité par Jean Rousset, Narcisse romancier, essai sur la première personne dans le roman, Paris, José Corti, 1972, p. 92).

88 JV21, t. I, p. 378-379; dimanche 2 juillet 1690.

89 Il s'inspire probablement du Discours à Mme de La Sablière de La Fontaine.

90 JV21, t. II, p. 115-117; samedi 25 novembre 1690. 
même genre que l'amène l'observation des chauves-souris : «Il y a des chauves-souris, qui méritent leur article. Elles sont faites comme les nôtres [...]. Je voudrais bien trouver un naturaliste qui me donnât une raison solide et convaincante pourquoi toutes les bêtes à quatre pieds : chevaux, ânes, bœufs, chiens, chats... et tous les autres voient clair la nuit aussi bien que le jour? [...] et pourquoi aussi il y en a, tel est l'homme, qui ne voient jamais clair par eux-mêmes, et ont besoin d'un secours étranger, du soleil, de la lune, des étoiles, ou d'un flambeau? Preuve encore de la sotte vanité de l'homme, et du peu de préférence que la nature lui donne sur les autres animaux». ${ }^{91}$

Chacun de ces épisodes nocturnes conduit invariablement Challe à des interrogations métaphysiques, comme si, cette fois, la solitude de la nuit sollicitait sa réflexion sur les questions existentielles.

D'autres notations du Journal de voyage renvoient à une expérience personnelle de la nuit en mer. Qu'il découvre un poisson exotique, Challe s'enthousiasme : "une dorade : c'est à mes yeux et à mon goût le plus beau et le meilleur poisson de la mer. On l'appelle dorade parce que ses écailles sont toutes dorées; et lorsque, dans une nuit sombre, telle qu'a été la dernière, ce poisson passe proche d'un vaisseau, on dirait d'une lame d'or». ${ }^{92}$ Pour décrire l'illumination nocturne de la mer sous l'équateur, il manifeste le même émerveillement : «La mer a paru presque toute la nuit éclatante en beaucoup d'endroits, et lumineuse partout, sans interruption, jusqu'à ce que le vent soit venu. On dit que cela arrive très souvent sous la Ligne, c'est-à-dire sous le soleil». ${ }^{93} \mathrm{Ce}$ pendant, s'il est sensible à la magie du spectacle que lui offrent les mers tropicales, il exprime ensuite son besoin d'explication en enregistrant les réflexions des missionnaires qui l'accompagnent avant de conclure : «mon esprit sceptique n'est point convaincu : il est toujours dans le doute». ${ }^{94}$ Systématiquement, les manifestations physiques de la nature entraînent un besoin d'explication qui se traduit par un refrain : je doute, je voudrais comprendre.

A l'instar du chevalier de Méré que la contemplation de la nuit entraînait à se poser des questions d'ordre métaphysique, ${ }^{95}$ Challe est

91 JV21, t. I, p. 378; dimanche 2 juillet 1690; la nature et non Dieu.

92 JV21, t. I, p. 247; vendredi 31 mars 1690.

93 JV21, t. I, p. 250 ; dimanche 2 avril 1690.

$94 J V 21$, t. I, p. 254; dimanche 2 avril 1690.

95 «Je trouve [...] que l'on void de plus grandes choses dans la retraite que dans le monde, ou du moins qu'on les y considère mieux [...]. Si nous regardons les div- 
sensible à l'étrangeté ou à la majesté du spectacle observé. Sa réflexion ou son inquiétude se fondent sur une observation précise : "La lune était dans son plein : elle a souffert une éclipse jusques à la moitié de son disque ; et cette éclipse a duré depuis son lever sur notre horizon jusques à ce qu'elle ait été dans le sud-est quart de sud, c'est-à-dire un peu plus de trois heures. Cela ne peut point avoir paru en France, parce que par la supputation des degrés de longitude, il ne pouvait être que onze heures et demie ou midi, au plus, de la journée d'hier...». ${ }^{96}$ Plus tard, la description du ciel nocturne éveille en lui une interrogation portant sur la situation de l'homme dans le monde qui rappelle l'angoisse pascalienne:

J'ai remarqué une chose cette nuit, environ sur les onze heures et demie. La lune dans son plein était justement au-dessus de notre tête ; et quoique ses rayons fussent à plomb et perpendiculaires, ils ne nous lançaient qu'une lumière fusque et sombre; au lieu qu'avant qu'elle fût à notre zénith ou après qu'elle l'a eu passé, sa lumière était belle et claire. Je voudrais bien savoir pourquoi ces rayons de la lune sont plus clairs obliques que perpendiculaires? Que de choses l'homme ignore! Ses sens sont frappés sans qu'il en comprenne la cause. Il se forme des raisons de tout : son amour-propre et son orgueil l'y clouent. Je crois $l^{\prime}$ avoir déjà dit ${ }^{97}$, je pardonnerais à l'homme de ne croire point ce qu'il ne voit pas s'il pouvait rendre raison de ce qu'il voit. ${ }^{98}$

ers ouvrages de la nature, le coucher du Soleil, une nuit tranquille et ces astres qui roulent si majestueusement sur nos testes, nous en sommes toûjours éstonnez. Ceux qui ne pensent qu'à leur fortune, sont occupez d'un petit monde artificiel qu'ils sçavent fort bien ; mais ce grand monde naturel leur est inconnu.» (Antoine Gombaud, chevalier de Méré, Troisième conversation, dans CEuvres complètes, éd. Charles-H. Boudhors, Paris, Fernand Roches, coll. «Les Textes français», 1930, t. I, p. 36.)

96 JV21, t. II, p. 61 ; mardi 19 septembre 1690.

97 Challe fait sans doute allusion à ce qu'il a dit à Charmot à la date du 8 mars 1690 : «La suite de la conversation m'engagea à dire à $\mathrm{M}$. Charmot que l'homme était bien malheureux, et en même temps bien orgueilleux, de vouloir, avec de faibles lumières, et aussi bornées que les siennes, monter et s'élever jusqu'à la connaissance de Dieu, qui est un Être incompréhensible, lui, qui ne se connaît pas luimême, et qui ne peut rendre aucune raison solide et convaincante des simples opérations de la nature, qui se passent dans lui-même et sous ses yeux, qui ne sont cependant que les moindres œuvres de la divinité» (JV21, t. I. p. 97).

JV21, t. II, p. 269 ; vendredi 16 février 1691. 
Mais lorsque Challe est au milieu de l'action, il n'est plus question de métaphysique. Rapportant la destruction d'un vaisseau anglais qu'ils détruisent en négligeant de le transformet en brûlot, Challe cite un vers de Scarron pour souligner l'effet magique produit par la nuit : «Le navire anglais que nous prîmes hier, et qui était de quelque trois cents tonneaux, aurait été métamorphosé en brûlot s'il avait été voilier ; mais n'allant point du tout, on y a mis le feu aujourd'hui. La flamme n'a rien d'affreux le jour : C'est dans l'obscurité, que la lumière est belle»."

Quand il dépeint le sabordage du Philip Harbert, il trouve d'emblée les mots pour décrire l'horreur et la fascination du spectacle. Dès la première version de son récit, le Journal à Pierre Raymond, tout est dit. Il reprendra le passage tel quel en 1721:

Sur les deux heures et demie après minuit, il se leva un petit vent de sud, dont il fit son profit autant qu'il put. Il mit toutes voiles dehors pour tâcher de nous échapper ; mais M. de Porrières, qui voulait lui donner ce matin le premier l'aubade, comme il lui avait donné la sérénade hier, a fait aventer ; et comme l'Ecueil va parfaitement bien, nous l'avons eu joint en peu de temps. Nous avions déjà cargué nos voiles, pour faire jouer nos violons, et attacher avec lui un combat réglé, et seul à seul, sous les voiles et à la mer, lorsqu'il a tiré le premier sur nous, et nous sur lui. A peine notre bordée a été lâchée que nous avons tout d'un coup entendu dans son entre-deux-ponts un bruit de mousqueterie lâchée comme d'un salut sans intermission. C'était un coffre plein d'artifice, qu'on nomme ordinairement coffre à feu.

Ce vaisseau parut tout d'un coup en feu et en flamme. Le désespoir de pouvoir le défendre avait obligé ce capitaine anglais à mettre lui-même le feu à son navire. Nous avons bien vu éloigner la chaloupe dans laquelle il s'est sauvé, mais nous l'avons bientôt perdue de vue. Nous nous sommes éloignés de ce navire le plus vite qu'il nous a été possible, crainte de quelque éclat qui aurait pu mettre le feu au nôtre.

Quelle horreur de voir un navire en feu! En un moment ce ne fut que flamme. Quelle horreur d'entendre les cris du reste de son équipage, que ce malheureux avait abandonné à une mort certaine! Quelle horreur d'entendre le mugissement des animaux, consumés tout en vie! Ce navire fut plus d'une heure et demie qu'il semblait un charbon ardent. Le feu qui sort de la fournaise n'est pas plus éclatant. Je ne crois pas qu'on puisse voir au monde pendant la nuit un spectacle plus horrible : surtout lorsque le feu eut pris aux poudres, il semblait

$99 J V 21$, t. II, p. 51 ; dimanche 27 août 1690. Les vers de Scarron sont tirés de Jodelet ou le maître valet, acte IV, sc. 3 . 
un enfer, qui vomissait feu et flamme contre le ciel. L'air en fut tout en feu pendant un demi-quart d'heure : ensuite succéda une noire et épaisse fumée, qui fut une grosse demi-heure à se dissiper.

C'est ainsi qu'a péri le Philip Harbert, de Londres, l'un des plus beaux et des plus forts navires qui fussent à la mer, et cela par l'intrépidité et l'inhumanité de son capitaine : digne assurément d'une meilleure fortune s'il eût suivi le parti de son prince ; mais homme à jamais condamnable, non seulement par cette raison mais aussi par la cruauté qu'il a eue d'abandonner aux flammes et à une mort également certaine et horrible les mêmes hommes qui avaient opiniâtrement secondé son courage et son désespoir. ${ }^{100}$

Une observation du même ordre, soulignant la magie de la nuit, se retrouve dans les Mémoires quand Challe évoque le désastre de La Hougue, le 29 mai 1690: "A l'égard du Prince dans lequel j'étais, n'entendant plus tirer du tout, nous mouillâmes par trente-deux brasses d'eau, le ciel couvert non pas de nuages, mais de la fumée de la prodigieuse quantité de coups qui avaient été tirés pendant la journée. Le temps s'éclaircit, et la lune qui était dans son plein nous fit voir que nous étions entourés de vaisseaux de toutes parts ${ }^{101}$ ". Puis, comme ils veulent fêter leur survie, Challe évoque ce surprenant banquet nocturne: «M. de Bagneux fit venir six bouteilles [de vin] au lieu d'une, et nous n'eûmes qu'une même table avec un pâté et un jambon dont chacun mangea avec appétit au clair de la lune. Pendant ce petit repas nocturne, Bonamy demanda à $M$. de Bagneux s'il ne voulait pas enterrer la synagogue avec honneur, c'est-à-dire périr plutôt que de se rendre». ${ }^{102}$

Challe aurait certainement souscrit au propos consigné par Diderot dans son Eloge de Richardson : "Vous avez vu cent fois le coucher du soleil, \& le lever des étoiles ; vous avez entendu la campagne retentir du chant éclatant des oiseaux; mais qui de vous a senti que c'étoit le bruit du jour qui rendoit le silence de la nuit plus touchant?» ${ }^{103}$

\section{JACQUES CORMIER}

Académie royale des Beaux-Arts de Bruxelles

$100 J V 21$, t. I, p. 404 et surtout p. 406-407; 3 juillet 1690

101 Mémoires, p. 271.

102 Id

103 Denis Diderot, Éloge de Richardson, dans Samuel Richardson, Lettres angloises, ou Histoire de Clarisse Harlove, Paris, Libraires associés, 1777, t. I, p. 25 ; nous soulignons. 
136 Jacques Cormier

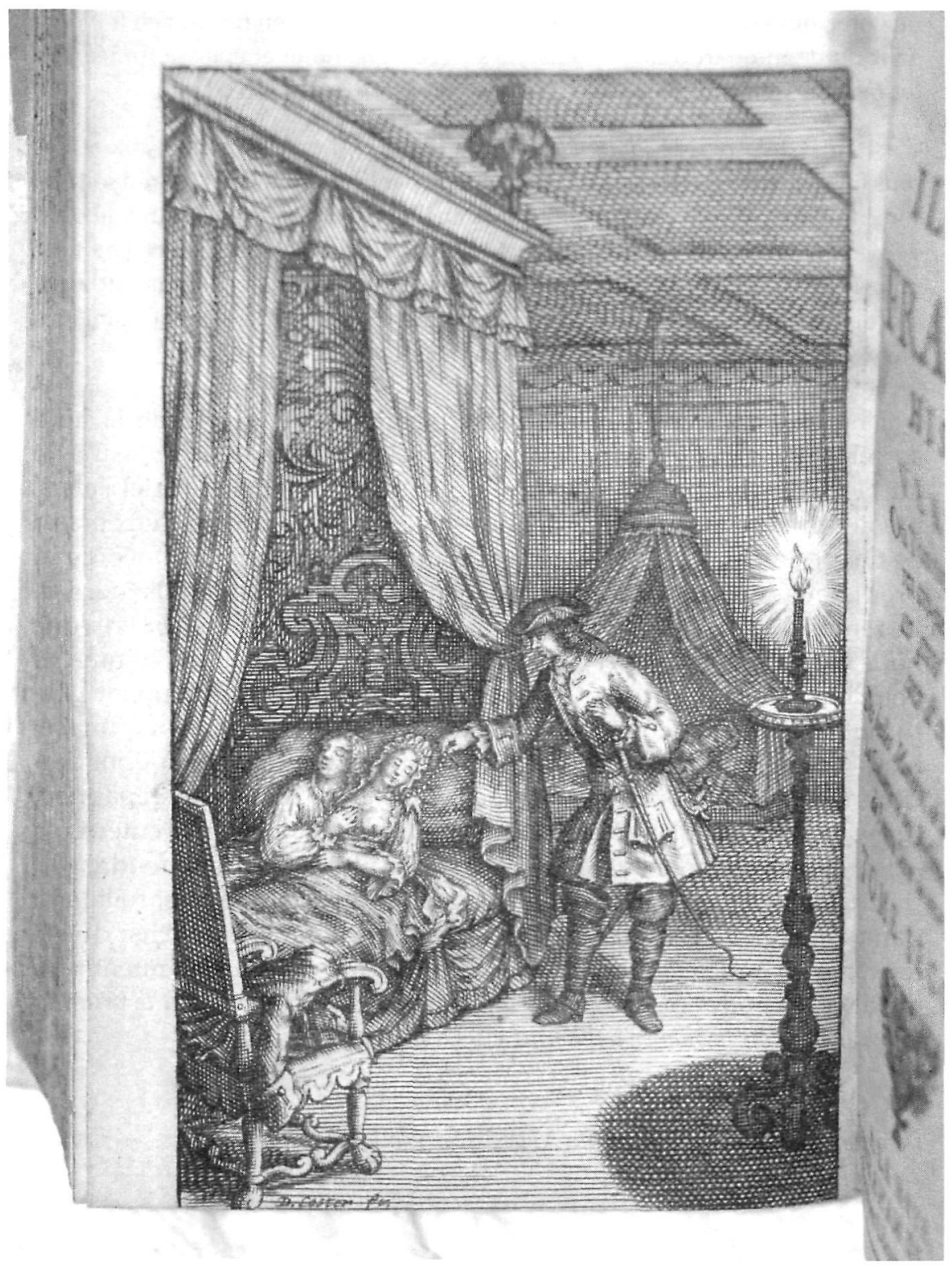

Figure 1 